\title{
Dynamically Reconfigurable Hybrid Cache: An Energy-Efficient Last-Level Cache Design
}

\author{
Yu-Ting Chen, Jason Cong, Hui Huang, Bin Liu, Chunyue Liu, Miodrag Potkonjak, and Glenn Reinman \\ Computer Science Department, University of California, Los Angeles \\ Los Angeles, CA 90095, USA \\ Email: \{ytchen, cong, huihuang, bliu, liucy, miodrag, reinman\}@cs.ucla.edu
}

\begin{abstract}
The recent development of non-volatile memory (NVM), such as spin-torque transfer magnetoresistive RAM (STT-RAM) and phase-change RAM (PRAM), with the advantage of low leakage and high density, provides an energy-efficient alternative to traditional SRAM in cache systems. We propose a novel reconfigurable hybrid cache architecture $(R H C)$, in which NVM is incorporated in the last-level cache together with SRAM. RHC can be reconfigured by powering on/off SRAM/NVM arrays in a way-based manner. In this work, we discuss both the architecture and circuit design issues for RHC. Furthermore, we provide hardware-based mechanisms to dynamically reconfigure RHC on-the-fly based on the cache demand. Experimental results on a wide range of benchmarks show that the proposed $\mathrm{RHC}$ achieves an average $63 \%, 48 \%$ and $25 \%$ energy saving over non-reconfigurable SRAM-based cache, non-reconfigurable hybrid cache, and reconfigurable SRAM-based cache. RHC only introduces at most $4 \%$ performance overhead over nonreconfigurable SRAM-based cache and non-reconfigurable hybrid cache.
\end{abstract}

\section{INTRODUCTION}

Traditional SRAM-based on-chip cache has become a bottleneck for energy-efficient design due to its high leakage power. Designers have turned their attention towards emerging non-volatile memories, such as the spin-torque transfer magnetoresistive RAM (STT-RAM) and phasechange RAM (PRAM), to build future memory systems. Power, performance, and density characteristics of the new technologies differ dramatically compared to SRAM, and thus they enlarge the landscape of memory design.

Table I shows a brief comparison of SRAM, STT-RAM, and PRAM technologies. The exact access time and dynamic power depend on the cache size and the peripheral circuit implementation. In sum, SRAM suffers from the high leakage and low density while providing great endurance; STT-RAM and PRAM provides high density and low leakage at the cost of weak enduarance. Morevoer, STT-RAM outperforms PRAM in terms of the access time and dynamic power, while PRAM has higher density.

TABLE I

COMPARISON AMONG SRAM, STT-RAM, PRAM.

\begin{tabular}{|c|c|c|c|}
\hline \hline & SRAM & STT-RAM & PRAM \\
\hline Density & $1 \mathrm{X}$ & $4 \mathrm{X}$ & $16 \mathrm{X}$ \\
\hline Read time & Very fast & Fast & Slow \\
\hline Write time & Very fast & Slow & Very slow \\
\hline Read power & Low & Low & Medium \\
\hline Write power & Low & High & High \\
\hline Leak. power & High & Low & Low \\
\hline Endurance & $10^{16}$ & $4 \times 10^{12}[1]$ & $10^{9}$ \\
\hline
\end{tabular}

With desirable characteristics on leakage power and density, NVMs have been explored as an efficient alternative for either SRAM or DRAM in memory systems [2][3][4][5][6]. As can be seen in Table I, compared to PRAM, STT-RAM has higher endurance $\left(10^{9}\right.$ versus $4 \times 10^{12}$ write cycles) [7][1]. Based on the write cycles, we use similar endurance model proposed in [8] to calculate the lifetime of PRAM and STT-RAM in a on-chip hybrid cache which consists of $1 \mathrm{MB}$ SRAM and 3MB NVM. Table II demonstrates the lifetime of three write-intensive workloads selected from medical imaging domain [9] and PARSEC [10]. For a PRAM-based hybrid cache, the lifetime is limited, which is from 4.70 to 196.12 days; but the STT-RAM-based hybrid cache can last for more than tens of years. Thus, STT-RAM is more suitable for on-chip last-level cache [2][3][4][5] design while PRAM is promising as an alternative of DRAM in the main memory design [6]. Therefore, in this paper, we will focus on a hybrid cache architecture with STT-RAM as the NVM.

TABLE II

ENERGY OF 4MB RHC AND 2MB SRAM-BASED CACHE

\begin{tabular}{|c|c|c|c|}
\hline \hline Workloads & registration & segmentation & fluidanimate \\
\hline PRAM (days) & 4.70 & 196.12 & 39.33 \\
\hline STT-RAM (years) & 12.88 & 537.32 & 107.76 \\
\hline
\end{tabular}

A common problem in the existing hybrid cache designs [2][3] is the lack of adaptation to varied workloads. Previous studies show that different applications may exhibit different characteristics [11]. For example, if the targeted applicaiton streamly access its working set, which is $10 \mathrm{MB}$, then a fixed hybrid cache design consisting of a $2 \mathrm{MB}$ SRAM and $8 \mathrm{MB}$ NVM (as discussed in [3]) may become inefficient in terms of both performance and energy compared to a 2MB SRAMonly design. In the 2MB SRAM-only design, all data blocks are put into the SRAM to achieve fast access. However, in the 2MB SRAM with $8 \mathrm{MB}$ NVM design, the data blocks are distributed in both the SRAM and NVM regions, while most blocks are located in the NVM region. The cache miss rate is the same for the two architectures due to streaming access pattern, but the performance degrades due to the longer access latency of NVM. The energy consumption in the hybrid design is larger since it consumes more leakage due to longer runtime and additional leakage from NVM arrays. Also, the higher dynamic write energy on NVM increases the total energy consumption. Therefore, if we can provide configurability on the hybrid cache design, it can be reconfigured to accommodate varied workloads. In this example, the hybrid cache can be reconfigured into 2MB SRAM to achieve the best performance.

In this paper, we propose a novel reconfigurable hybrid cache design $(R H C)$. Our design explores the use of NVM to partially replace SRAM in the last-level cache to efficiently reduce leakage energy. The proposed RHC design supports reconfigurable SRAM/NVM size, with the capability of powering on/off SRAM and NVM arrays in a way-based manner for better accommodation of memory requirements from different workloads. Hardware-based mechanisms are proposed to detect the cache demand for dynamic reconfiguration. On average, RHC signfincantly saves $64 \%, 46 \%$ and $28 \%$ energy over non-reconfigurable SRAM cache, non-reconfigurable hybrid cache and reconfigurable SRAM cache, respectively. Meanwhile, only little performance overhead (less than 4\%) are introduced when compared to non-reconfigurable SRAM cache and non-reconfigurable hybrid cache. 


\section{RELATED WORK}

Because of the desirable characteristics on leakage power and density, NVMs have been intensively inversitaged recently as an efficient alternative for either SRAM in the on-chip caches or DRAM in the main memory [2][3][4][5][6]. In [2], STT-RAM and PRAM are positioned into lower-level cache. Two types of hybrid cache architectures are evaluated - inter-level and intra-level, in which NVMs are utilized either as the entire L3 cache or the slow-accessed region in L2 cache. In [3], 3D stacking STT-RAM is used to build a hybrid cache system with SRAM. However, none of the prior works have considered dynamic powering on/off SRAM and NVM arrays to adapt to varied workloads.

Dynamic reconfigurable caches are invesigated for the pure SRAM cache to either reduce the energy consumption through power gating [12][13][14][15][16], or provide dynamic flexible support of software-managed memories to the core through cache line control bit [17][18]. The key of these approaches is the dynamic assessment of cache pressure. In [12], researchers use a single miss counter to measure the demand of an instruction cache to perform reconfiguration. Missing tags or victim tags are used in [13][14][18] to assess the cache pressure. When a cache miss occurs, the tag of the victim block will overwrite the LRU tag in the same set in victim tags and be marked as MRU victim tag. If there is a cache miss and victim tags hit, this indicates that a potential hit will occur if the requested block is held in the cache. The authors in [15] use a time-based counter for each cache block, which will be reset once there is a hit to that block. Once the counter exceeds a given decaying period, the block will be turned off to save leakage. However, none of the existing dynamic reconfiguration schemes have considered hybrid memory technologies.

To the best of our knowledge, this paper is the first work to explore dynamic cache reconfiguration for hybrid memory technologies in order to reduce the cache energy comsumption.

There are also works investigating the endurance reduction for the NVMs. In [6][19], wear-leveling techniques are proposed for a PRAM-based memory system to enhance the lifetime. Recent work in [1] uses periodically set-remapping to distribute the writes among sets in a STT-RAM cache. Another set of work migrates the writeintensive cache blocks to other cache lines in the same/different cache set or in the SRAM to reduce the average write frequency of the STTRAM (or PRAM) cache lines [8]. These works are orthogonal and complementary to our proposed reconfigurable hybrid cache designs.

\section{RECONFIGURABLE HYBRID CACHE DESIGN}

In this section we discuss the RHC design in the following way. First, we present the support of architecture and circuit design for RHC with disparate SRAM and NVM technologies. Next, the reconfigurability support for RHC is discussed. At last, the hardwarebased dynamic reconfiguration scheme is demonstrated.

\section{A. Hybrid Cache Architecture}

Figure 1 shows an overall diagram of RHC. In RHC the data array is partitioned into SRAM and NVM at a cache-way granularity. One concern of RHC design is that the access latency of a NVM cell is longer than that of SRAM [2]. Considering a simple hybrid cache design in which the tag and data arrays of each cache way are implemented either with all SRAM cells or NVM cells, the cache critical path in such a design will always be dominated by the longer access latency to the NVM cache ways. To overcome this, RHC is designed in the following way. First, the accesses to the tag array and data array are conducted in a sequential style, which means the data array will be accessed after the tag array. Such a serialized tag/data array access has already been widely adopted in a modern low-level large-scale cache for energy reduction. Second, the RHC tag array is fully implemented with SRAM cells. In RHC, each tag entry contains only four bytes, including the tag, coherence state bits, and the dirty bits, etc., while each cache block in the data array contains 64 bytes. Hence, the SRAM-based RHC tag array will not create a large energy overhead.

The circuit design of RHC with STT-RAM as the NVM is as follows. First, a STT-RAM cell has a bitline (BL) and a source-line (SL) for its operation. This is similar to the bitlines (BL, BLB) used in SRAM. Therefore, the organization of a STT-RAM data array is almost the same as a SRAM data array. Second, the sense amplifiers need to be modified due to the single-ended bitlines [4]. According to a recent implementation [20] of a STT-RAM array, the reference voltage is $1.2 \mathrm{~V}$, which is close to a SRAM-based design. Therefore, additional pins for power supply may not be required, and the VPCX, VREAD, and VWRITE can be removed to simplify circuit design.

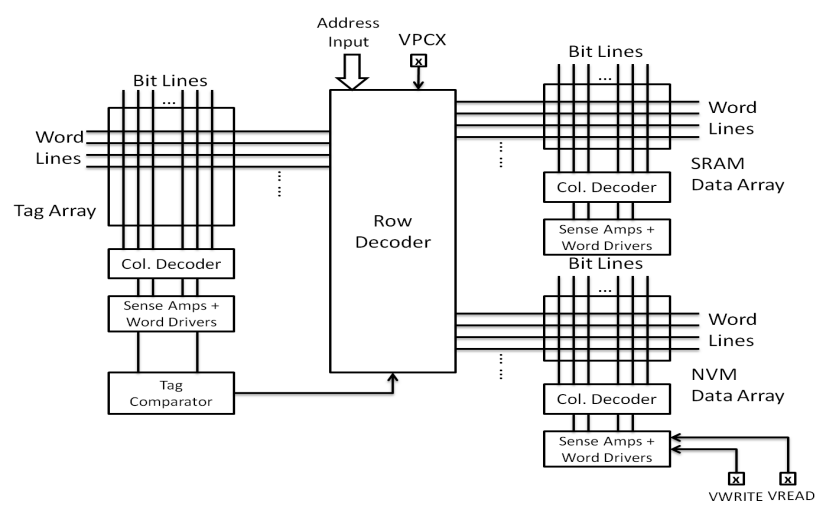

Fig. 1. Reconfigurable hybrid cache (RHC) design

\section{B. RHC Reconfiguration Design}

The reconfiguration in RHC is realized by powering on/off SRAM and NVM arrays arbitrarily in a way-based manner. From an architectural point of view, the reconfiguration mechanism in RHC is similar to the existing way-based reconfigurable SRAM cache [21]. Data access will not be directed to a disabled cache way, thus those ways in the data array dissipate no dynamic power. Note that the replacement decision logic within the cache controller must ensure that no data will be allocated to a disabled cache way.

In Figure 2 we illustrate the power-gating design adopted in RHC. A centralized power management unit $(P M U)$ is introduced to send sleep/wakeup signals to power on/off each SRAM or NVM way. The power-gating circuits of each way in SRAM tag/data arrays are implemented with NMOS sleep transistors to minimize the leakage. In this design the stacking effect of three NMOS transistors from the bitline to GND substantially reduces leakage [12]. Note that in RHC the SRAM cells in the same cache way will be connected to a shared virtual GND while the virtual GNDs among different cache ways are disconnected. This can ensure that the behaviors of cache ways that are powered-on will not be influenced by the powering-off process in other ways.

For the peripheral circuits, such as row decoder, column decoders, word drivers, and sense amplifiers, we use PMOS sleep transistors to implement the power-gating design; this can provide better performance of the peripheral circuits in the active mode [16]. Since the NVM cell itself consumes little leakage, we do not introduce extra power-gating circuits for the cells of NVM data arrays. To power on/off a NVM cache way, PMU will send a sleep/wakeup control signal to the peripheral circuits of the corresponding NVM way. The design complexity of PMU is highly related to the adopted wakeup scheme. In this work we assume a daisy-chain wakeup scheme for each cache way [22]. For PMU, we use Synopsys SAED 90nm technology, which is the most advanced process technology available, 
to obtain the energy and delay number. A RTL-level description of PMU is synthesized by Synopsys Design Compiler ${ }^{T M}$. The dynamic energy is $0.0135 \mathrm{pJ}$ for one reconfiguration, while the leakage power is $1.0378 \mathrm{uA}$. The delay of PMU is $0.28 \mathrm{~ns}$. The overhead of PMU is relatively small and thus can be neglected.

The overhead of the reconfiguration will be classified in the following two categories. First, when a cache way is disabled, the dirty blocks in that cache way need to be written back to lower-level memory. This will introduce both performance and energy overhead. Second, from a circuit-level perspective, the power-up process also involves extra energy consumption. The reason for this is that the accumulated charge during the standby mode in SRAM cells should be discharged.

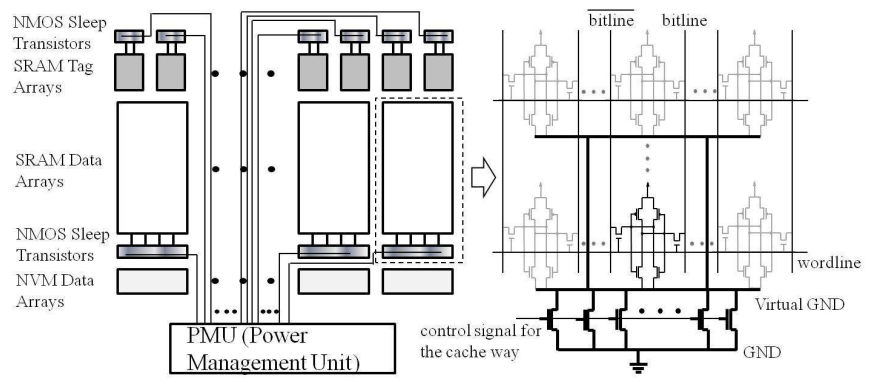

Fig. 2. Power-gating design for RHC

\section{Dynamic Reconfiguration}

In this section, we propose two hardware schemes to utilize the reconfigurability provided by RHC. The main idea is to detect the cache demand dynamically and thus RHC can be reconfigured in a way-based manner to satisfy the demand. In the meantime, the powered-off cache ways provide energy saving in leakage.

1) Way-Based Decay Scheme: The reconfiguration scheme includes two dynamic decisions: (1) when to power off a cache way and (2) when to power on a cache way. To power off a cache way, we utilize the cache decay idea [15], and introduce the novel way-based decay counters, as shown in Figure 3. The main idea of cache decay is to power off a cache block which is not accessed for a long time period to save leakage. This time period is called the decay interval. Cache decay is implemented by a local 2-bit saturating counter for each block with a global counter. The local counter is incremented when the global counter exceeds a certain number of clock cycles, which is used to model the decay interval. The local counter is reset to zero when there is an access on this block. Cache decay is initially used to provide a self-guided block-based power-on/off mechanism [15]. However, it is not feasible for PMU to arbitrate reconfiguration in the block-based granularity due to the intolerable circuit design complexity. Therefore, we use the way-based decay counter to measure the number of decay blocks in that cache way during a time period. The way-based decay counter increments by one when any local 2-bit counter in that cache way saturates. Similarly, when a local 2-bit counter is reset to zero, the corresponding waybased decay counter decreases by one. If the value of a way-based decay counter exceeds a given threshold in a given time period, such as $90 \%$ (used in this work) of the blocks in that way, the whole cache way will be powered off due to the low cache demand.

To detect the demand for powering on more cache ways, we keep the whole tag array powered-on to record potential hits if those blocks are in RHC. The potential hit counter increments by one when a hit occurs on a tag entry whose corresponding data block is poweredoff. This is similar to the VTB counters used in [14][18]. The update of the potential hit counter reuses the same tag array, which does not create extra storage overhead. The replacement policy for the tags of the powered-off blocks follows LRU policy. When the value of potential hit counter is greater than a threshold, a cache way is powered on to reduce cache misses. We denote this powering-on threshold as $T H_{\text {on }}$. Note that the power-on/off decision is made for every one-million cycles in this work. This time period is called reconfiguration period. Both the way-based decay counters and the potential hit counter are reset to zero after the decision is made.

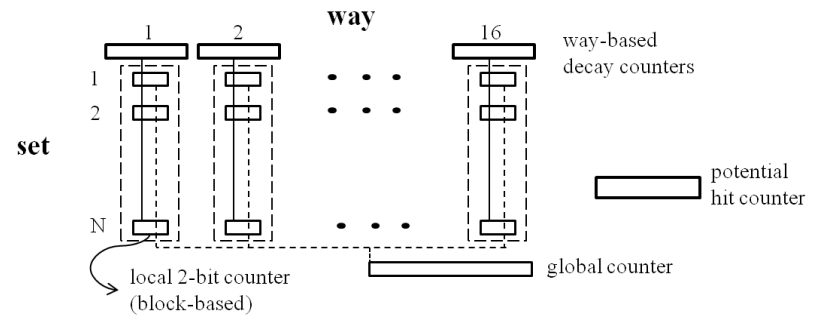

Fig. 3. Counters for dynamic reconfiguration

2) Independent Potential Hit Counters Scheme: In this section, we provide an improved strategy to for dynamic reconfiguration. In the way-based decay scheme, a large number of cache ways can be powered off simultaneously since each cache way is controlled independently. However, we observed that this aggressive poweringoff scheme results in significant performance degradation due to the increase of L2 cache misses especially when the decay interval is small, such as one million cycles. Another potential problem is that a single decay interval cannot accurately capture varied decay intervals of all cache blocks, which also makes the way-based decay counter ineffective. When the decay interval is too large, such as 100 millions cycles, most of the blocks are accessed once during that interval. The powering-off decisions are seldomly made and thus the energy reduction is limited.

The improved scheme takes both of the hybrid nature of RHC and the aggressive powering-off issue into consideration. Considering the hybrid nature of RHC, it is beneficial to measure the cache demand for the SRAM and STT-RAM array independently to better accommodate the cache demand. Therefore, we use two potential hit counters to measure the cache demand of the SRAM and STT-RAM arrays independently.

The powering-off strategy is different from that of the waybased decay scheme. Here, we use the same potential hit counter to make the powering-off decision. We introduce another poweringoff threshold $\left(T H_{o f f}\right)$. When the value of the potential hit counter is less than or equal to $T H_{o f f}$, a cache way can be powered off. Based on the strategy, only one cache way can be powered off at a time period, and this greatly reduces the chance of cache threshing. However, according to our observation, this strategy still generates considerable cache misses. To mitigate the aggressive powering-off strategy, we further restrict the powering-off condition. When the value of the potential hit counter reaches $T H_{o f f}$, we cannot power of a cache way immediately. A cache way can only be powered off until ten consecutive reconfiguration periods (ten-million cycles) are waited. Note that $T H_{o n}$ is set to 50 and $T H_{o f f}$ is set to 0 for both SRAM and STT-RAM arrays for evaluation.

Furthermore, we consider the endurance of RHC when making decisions of reconfiguration. We achieve this by randomly selecting the cache way from all possible candidates. For example, when the decision is to power off a cache way, we will randomly pick the victim from all powered-on cache ways.

\section{Evaluation Methodology}

\section{A. Performance and Energy Models}

We evaluate the proposed RHC design on a simulation platform built upon Simics [23] with GEMS [24]. Table III shows the parameters used in our model. The value $K$ represents the number of 
cache ways that are powered on in a specific L2 cache configuration, which also equals the amount of "active" cache associativity. Notice that the configuration of the processor core, L1 caches, and main memory remains the same through all simulations.

TABLE III

SIMULATION PARAMETERS

\begin{tabular}{|c|c|c|}
\hline & single-thread workload & multi-thread workload \\
\hline \#Core & 1 & 4 \\
\hline Core & \multicolumn{2}{|c|}{ Sun UltraSPARC-III $\mathrm{Cu}$ processor core, $4 \mathrm{GHz}$} \\
\hline L1 Cache & \multicolumn{2}{|c|}{$\begin{array}{l}32 \mathrm{~KB} \text { per core for I/D caches } \\
4 \text {-way, } 64 \text {-byte block, } 1 \text {-cycle latency }\end{array}$} \\
\hline L2 Cache & \multicolumn{2}{|c|}{$\begin{array}{l}\text { RHC: 1MB SRAM + 3MB STT-RAM } \\
\text { SRAM-based: } 2 \mathrm{MB} \\
K \text {-way }(\mathrm{K} \leq 16), 64 \text {-byte block }\end{array}$} \\
\hline $\begin{array}{l}\text { L2 Cache } \\
\text { Access Lat. }\end{array}$ & \multicolumn{2}{|c|}{$\begin{array}{l}\text { STT-RAM read: } 11 \text { cycles } \\
\text { STT-RAM write: } 30 \text { cycles }\end{array}$} \\
\hline Main Memory & \multicolumn{2}{|c|}{ 4GB, 320-cycle access latency } \\
\hline
\end{tabular}

For the energy of the memory technologies, we use the ITRS $32 \mathrm{~nm}$ process model. The SRAM and STT-RAM energy/latency numbers used in our simulations are obtained from CACTI 6.5 [25] and the data scaled from [4], respectively. The energy numbers of a 4MB RHC and 2MB SRAM-based cache are listed in Table IV, where Active and Standby correspond to the power-on and poweroff state. The standby leakage is estimated according to the ratio of active/standby leakage presented in [12]. This can be achieved through a careful power-gating design.

TABLE IV

ENERGY OF 4MB RHC AND 2MB SRAM-BASED CACHE

\begin{tabular}{|c|c|c|c|c|}
\hline \hline $\begin{array}{c}\text { L2 Cache } \\
\text { Design }\end{array}$ & Tech. & $\begin{array}{c}\text { Dyn. energy } \\
\text { per acc. (nJ) }\end{array}$ & $\begin{array}{c}\text { Active } \\
\text { leak.(mW) }\end{array}$ & $\begin{array}{c}\text { Standby } \\
\text { leak.(mW) }\end{array}$ \\
\hline 4MB RHC & SRAM & 0.137 & 431.30 & 14.38 \\
\cline { 2 - 5 } & STT- & Read: 0.278 & 116.92 & 3.897 \\
\cline { 3 - 4 } & RAM & Write: 0.765 & & \\
\hline 2MB SRAM & & 0.288 & 711.29 & 23.71 \\
\hline
\end{tabular}

\section{B. Benchmarks}

Our testbenchs consist of 16 benchmark applications, which have been carefully chosen to represent memory intensive algorithms in the fields of data processing, massive communication, scientific computation and medical applications. The applications include seven memory-intensive applications from SPEC2006 [26], four applications from PARSEC [10], and five applications from the medical imaging domain [9].

TABLE V

WORKLOADS

\begin{tabular}{|c|c|}
\hline Benchmark & Applications \\
\hline SPEC2006 & $\begin{array}{l}\text { bzip2, mcf, soplex, libquantum, } \\
\text { h264ref, lbm, astar }\end{array}$ \\
\hline PARSEC(simmedium) & $\begin{array}{l}\text { blackscholes, swaptions, fluidanimate, } \\
\text { bodytrack }\end{array}$ \\
\hline Med. Imaging & $\begin{array}{l}\text { rician-denoise, gaussian-deblure, registration, } \\
\text { segmentation, compressive sensing }\end{array}$ \\
\hline
\end{tabular}

\section{Reference Designs}

To evaluate the effectiveness of RHC, we compare RHC with a traditional SRAM-based cache under the same area basis. RHC is set to $4 \mathrm{MB}$, which is composed of $1 \mathrm{MB}$ SRAM and 3MB STT-RAM, while the SRAM-based cache is set to $2 \mathrm{MB}$. This setting reflects the fact that STT-RAM is about four times denser than that of SRAM.
The area of the data arrays in $4 \mathrm{MB}$ RHC is about $0.875 \mathrm{X}$ that of the 2MB SRAM-based cache.

The associativity of both the 4MB RHC and 2MB SRAM-based cache are both 16-way. This setting provides the same reconfigurability on RHC and SRAM-based cache. RHC has four SRAM ways and 12 STT-RAM ways, while the SRAM-based cache has 16 SRAM ways. Both can be reconfigured from one cache way to 16 cache ways. To evaluate the effectiveness of RHC, we compare the performance and energy of RHC with three reference points: (1) $S C$ : non-reconfigurable $2 \mathrm{MB}$ SRAM-based cache; (2) $H C$ : nonreconfigurable $4 \mathrm{MB}$ hybrid cache (4-way SRAM + 12-way STTRAM); and (3) RSC: reconfigurable 2MB SRAM-based cache. Note that the evaluation in Section V-A and Section V-C utilizes the scheme introduced in Section III-C2 for both RHC and RSC while RSC uses a single potential hit counter.

\section{RESULTS}

\section{A. Effectiveness of RHC}

Figure 4 shows the comparison results of L2 cache miss rate. Compared to the baseline SC, $\mathrm{HC}$ consistently has lower miss rate (39\% on average) because of the $2 \mathrm{X}$ larger cache capacity provided by the STT-RAM. But this consistent miss rate improvment is realized at the cost of more energy consumption, which will be discussed later. On the other hand, since RSC dynamically powers off the cache ways - although this is done based on the cache pressure - the reduced cache capacity consistently impairs the cache performance $(77 \%$ more cache misses), especially when the dynamic reconfiguration scheme can not accurately capture the cache behaviour, This can be observed in bzip2 and libquantum. Compared to the baseline SC, the miss rate of RHC has two situations: 1) for the cases where the applications have relatively large working set (such as bzip2, deblure and compressive sensing), RHC can achieve considerable miss rate reduction of $52 \%$ on average; 2) for the cases where the applications have relatively small working set which can be hold for a 2MB L2 cache, RHC will gradully power off half of the cache capacity. But during this process, some of the cache blocks with long reuse distance will be evicted which results in slightly higher miss rate. In sum, RHC incurs $33 \%$ more cache misses compared to SC.

Figure 5 shows the comparison results of system performance in terms of runtime of the application on the system. These results are normalized to that of the baseline SC scheme. The runtime difference of the four design schemes mainly comes from the difference of the L2 cache miss rate. Compared to the baseline SC. HC consistently has better performance ( $0 \%$ to $36 \%$ less runtime) because of its consistently smaller miss rate, while RSC consistenly has worse performance ( $0 \%$ to $9 \%$ more runtime) due to its consistently larger miss rate. For the cases where RHC can achieves considerable L2 miss rate reduction, it also improves the performance (1\% to $34 \%$ less runtime) over SC. For the other cases, RHC incurs a slighly performance overhead ( $0 \%$ to $4 \%$ worse runtime).

As it comes to energy, the power of the dynamic reconfiguration begins to show gain. Figure 5 shows the comparison results of memory subsystem energy, The energy data is broken down into the L1 cache dynamic/leakage energy, the L2 cache SRAM/STTRAM dynamic/leakage energy for detail illustration of the energy distribution. These results are normalized to that of the baseline SC scheme. As can be seen that, the SRAM leakage dominates the memory subsystem energy in the $32 \mathrm{~nm}$ technology. Compared to the baseline SC, HC reduces energy by $24 \%$ to $53 \%$ (30\% on average), because the STT-RAM array consume less leakage. In addition, HC consistently reduces the runtime, which reduces the SRAM leakage. By dynamically powering off the cache ways based on cache pressure, RSC can also reduce the energy by $7 \%$ to $88 \%$ (51\% on average). In cases of bzip2, deblure and compressive sensing where the poweringon time of the remaining cache ways incurs a energy overhead which 


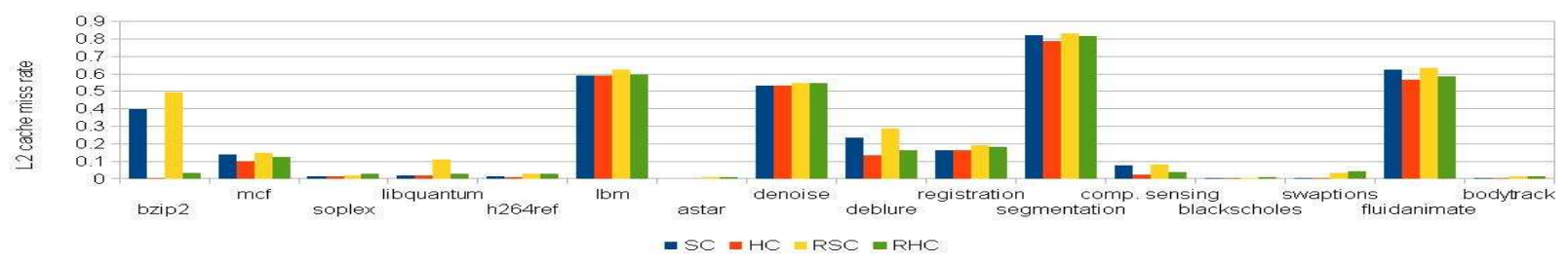

Fig. 4. Comparison results of $\mathrm{L} 2$ cache miss rate

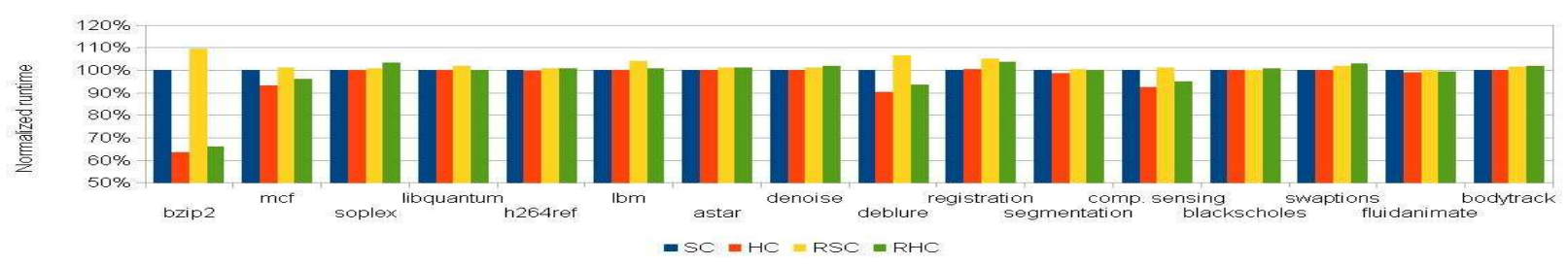

Fig. 5. Comparison results of runtime

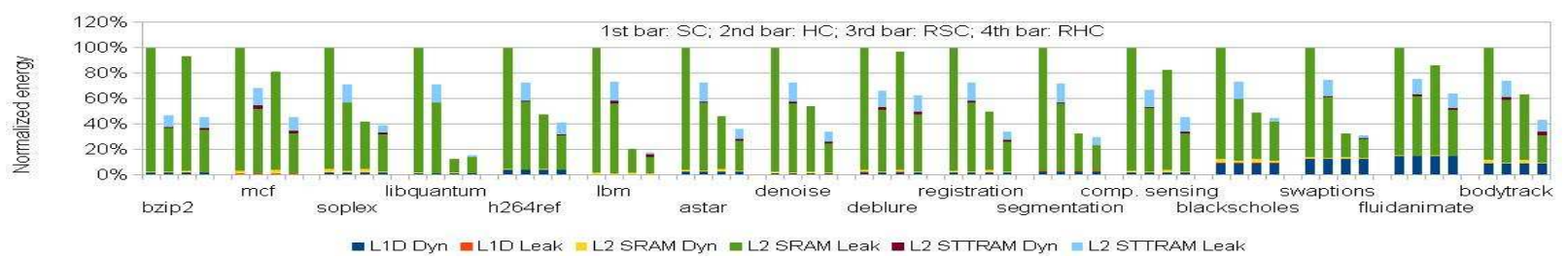

Fig. 6. Comparison results of memory subsystem energy

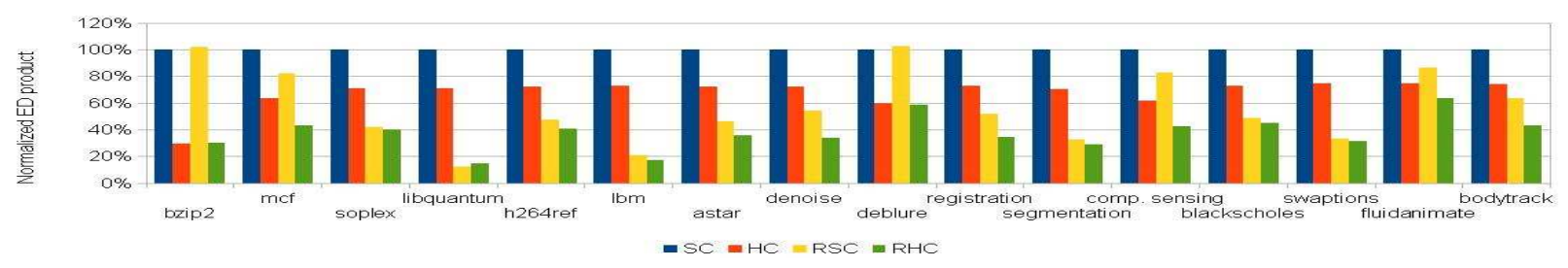

Fig. 7. Comparison results of ED product

almost catches up with the reduction of the leakage in the poweringoff cache ways, the energy reduction RSC has an energy overhead is much smaller than the other cases. By dynamically powering off the cache ways and maintaining the system performance, RHC achieves the least energy among all the design schemes since RHC inherits both advantages of the low leakage NVM array and dynamic reconfiguration to save leakage. It reduces energy by $63 \%, 48 \%, 25 \%$ compared to baseline SC, RSC and HC, respectively.

To better illustrate the gain over other design schemes in terms of both energy and runtime, we use the metrics of energy-delay product (ED) to make the comparison, where the delay means the runtime. Figure 7 shows the comparison results of this metric over the four design schemes. All results are normailzed to that of the baseline SC. As can be seen that, the prospoed RHC achieves the best among all the design schemes. On average, RHC improves the ED by $64 \%$, $46 \%$, and $28 \%$ compared to SC, HC and RSC, repspectively.

\section{B. Comparison of Two Dynamic Schemes}

For the way-based decay scheme, we evaluate on three different decay intervals $(1 \mathrm{M}, 10 \mathrm{M}$, and $50 \mathrm{M}$ cycles). When the decay interval is larger, more cache ways are powered-on to maintain the performance. The largest interval we used is $50 \mathrm{M}$ cycles since the simulation results remains the same even when we enlarge the decay interval. IPHC stands for the scheme using independent potential hit counters. Figure V-B shows the comparison results of runtime and energy. The results are normalized to the baseline HC. The most critical disadvantage of the way-based decay scheme comes from the significant performance degradation, as shown in Figure $8(\mathrm{a})$. When the decay interval is set to $1 \mathrm{M}$ cycles, the performance degradation are from $2 \%$ to $131 \%$ compared to HC. Even when the decay interval is set to $50 \mathrm{M}$ cycles, swaptions still suffers from $27 \%$ performance degradation. In constrast, IPHC can provide stable performance within $4 \%$ degradation compared to $\mathrm{HC}$ among all workloads.

Figure 8(b) shows the comparison of energy. IPHC can achieve better or at least similar energy reduction compared to the cases of $10 \mathrm{M}$ and $50 \mathrm{M}$ decay intervals. Therefore, IPHC can further provide energy saving when maintaining similar performance compared to the $50 \mathrm{M}$ decay interval case. When the decay interval is set to $1 \mathrm{M}$ cycles, the way-based decay scheme achieves much better energy saving on bzip2, segmentation, and comp.sensing. However, bzip 2 and comp.sensing suffer from $122 \%$ and $24.2 \%$ performance overhead compared to IPHC. In summary, IPHC provides consistent performance compared to baseline $\mathrm{HC}$ while providing considerable energy saving. Way-based decay scheme suffers from potential performance degradation problem and the choice of a suitable decay interval varies 
from workloads.

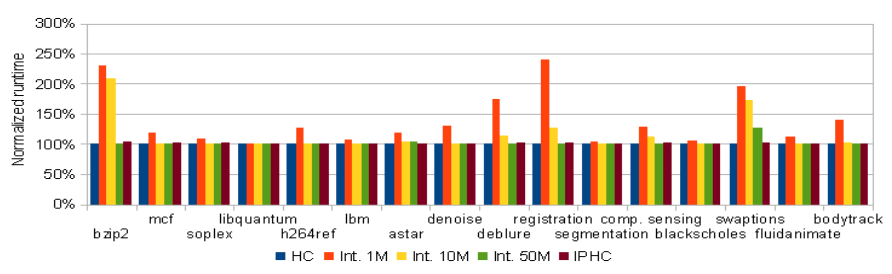

(a) Runtime

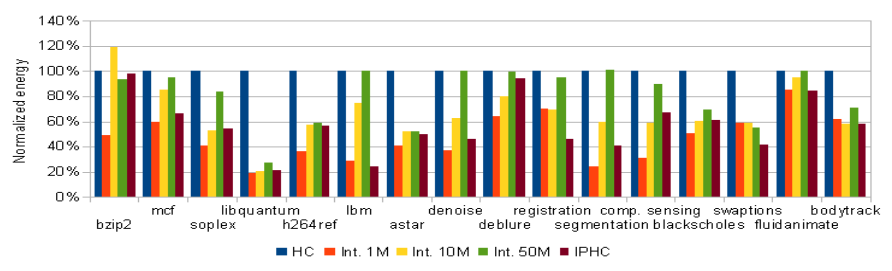

(b) Energy consumption

Fig. 8. Comparison of runtime, energy, on two dynamic schemes

\section{Endurance Analysis}

Table VI shows the endurance comparison between HC and RHC. The lifetime calculation is based on the method from [8] and the write cycles is $4 \times 10^{12}$ [1]. RHC can achieve from $1.08 \mathrm{X}$ to $3.53 \mathrm{X}$ lifetime enhancement from most of the workloads through random selection of powered-on/off ways except bzip2, soplex, segmentation, and swaptions. For bzip2 and segmentation, the lifetimes of our scheme are still in a reasonable range. Therefore, our reconfigurable scheme can achieve reasonable lifetime compared to $\mathrm{HC}$ even when available cache ways are limited due to reconfiguration. However, we observe the non-uniform distribution of write accesses as mentioned in the previous work [8][1]. Therefore, a suitable wear-leveling technique is still required to achieve better endurance.

TABLE VI

ENDURANCE COMPARISON 4MB NON-RECONFIGURABLE HYBRID CACHE (HC) AND 4MB RHC (UNIT: YEAR)

\begin{tabular}{|c|c|c||c|c|c|}
\hline \hline Workloads & HC & RHC & Workloads & HC & RHC \\
\hline bzip2 & 299.92 & 200.24 & g.-deblure & 76.68 & 116.36 \\
\hline mcf & 8.40 & 29.68 & registration & 12.88 & 30.68 \\
\hline soplex & 4.64 & 4.6 & segmentation & 537.32 & 256.4 \\
\hline libquantum & 2.82 & 4.2 & comp. sensing & 3.28 & 3.56 \\
\hline h264ref & 22.76 & 41.88 & blackscholes & 3.144 & 5.44 \\
\hline lbm & 228.96 & 253 & swaptions & 7.36 & 3.4 \\
\hline astar & 16.00 & 30.08 & fluidanimate & 107.76 & 118.56 \\
\hline r.-denoise & 53.44 & 118.8 & bodytrack & 9.76 & 10.28 \\
\hline
\end{tabular}

\section{CONCLUSIONS}

We propose an energy-efficient last-level cache design - reconfigurable hybrid cache (RHC). In RHC different memory technologies (SRAM and NVM) are unified at the same cache level to form a hybrid design, and power gating circuitry is introduced to allow adaptive powering on/off of SRAM/NVM sub-arrays at way level. With the proposed dynamic reconfiguration mechanism, RHC can provide $64 \%, 46 \%$ and $28 \%$ energy-delay product gains over nonreconfigurable SRAM-based cache, non-reconfigurable hybrid cache and reconfigurable SRAM-based cache, respectively. In the meantime, RHC can maintain the lifetime compared to the baseline, nonreconfigurable cache.

\section{ACKNOWLEDGEMENTS}

This work is partially supported by the SRC Contract 2009TJ-1984, and the Center for Domain Specific Computing (NSF Expedition in Computing Award CCF-0926127).

\section{REFERENCES}

[1] Y. Chen, W.-F. Wong, H. Li, and C.-K. Koh, "Processor caches built using multi-level spin-transfer torque ram cells," in Proc. ISLPED 2011 2011, pp. 73-78.

[2] X. Wu, J. Li, L. Zhang, E. Speight, R. Rajamony, and Y. Xie, "Hybrid Cache Architecture with Disparate Memory Technologies," in Proc. ISCA, 2009, pp. 34-45.

[3] G. Sun, X. Dong, Y. Xie, J. Li, and Y. Chen, "A Novel Architecture of the 3D Stacked MRAM L2 Cache for CMPs," in Proc. HPCA, 2008, pp. 239-249.

[4] X. Dong, X. Wu, G. Sun, Y. Xie, H. Li, and Y. Chen, "Circuit and Microarchitecture Evaluation of 3D Stacking Magnetic RAM (MRAM) as a Universal Memory Replacement," in Proc. DAC, 2008, pp. 554-559.

[5] M. Rasquinha, D. Choudhary, S. Chatterjee, S. Mukhopadhyay, and S. Yalamanchili, "An Energy Efficient Cache Design Using Spin Torque Transfer (STT) RAM," in Proc. ISLPED, 2010, pp. 389-394.

[6] B. Lee, E. Ipek, O. Mutlu, and D. Burger, "Architecting Phase Change Memory as a Scalable DRAM Alternative," in Proc. ISCA, 2009, pp. $2-13$.

[7] International Technology Roadmap for Semiconductors (ITRS) Website, http://www.itrs.net/Links/2009ITRS/Home2009.htm.

[8] A. Jadidi, M. Arjomand, and H. Sarbazi-Azad, "High-endurance and performance-efficient design of hybrid cache architectures through adaptive line replacement," in Proc. ISLPED, 2011, pp. 79-84.

[9] A. Bui, K.Cheng, J. Cong, L. Vese, Y. Wang, B. Yuan, and Y. Zou, "Platform Characterization for Domain-Specific Computing," in Proc. ASPDAC, 2012.

[10] C. Bienia, S. Kumar, J. P. Singh, and K. Li, "The PARSEC Benchmark Suite: Characterization and Architectural Implications," in Proc. PACT, 2008, pp. 72-81.

[11] P. Ranganathan, S. Adve, and N. P. Jouppi, "Reconfigurable Caches and their Application to Media Processing," in Proc. ISCA, 2000, pp. 214 224.

[12] M. Powell, S. Yang, B. Falsafi, K. Roy, and T. N. Vijaykumar, "GatedVdd: A Circuit Technique to Reduce Leakage in Deep-Submicron Cache Memories," in Proc. ISLPED, 2000, pp. 90-95.

[13] M. Zhang and K. Asanovic, "Fine-grain CAM-tag cache resizing using miss tags," in Proc. ISLPED, 2002, pp. 130-135.

[14] H. Zhou, M. C. Toburen, E. Rotenberg, and T. M. Conte, "Adaptive mode control: A static-power-efficient cache design," ACM Trans. Embed. Comput. Syst., pp. 347-372.

[15] S. Kaxiras, Z. Hu, and M. Martonosi, "Cache Decay: Exploiting Generational Behavior to Reduce Cache Leakage Power," in Proc. ISCA, 2001, pp. 240-251.

[16] J. Chang, M. Huang, J. Shoemaker, J. Benoit, S.-L. Chen, W. Chen, S. Chiu, R. Ganesan, G. Leong, V. Lukka, S. Rusu, and D. Srivastava, "The 65-nm 16-MB Shared On-Die L3 Cache for the Dual-Core Intel Xeon Processor 7100 Series," in JSSC, 2007, pp. 846-852.

[17] D. Chiou, P. Jain, L. Rudolph, and S. Devadas, "Applicationspecific Memory Management for Embedded Systems Using SoftwareControlled Caches," in Proc. DAC, 2000, pp. 416-419.

[18] J. Cong, K. Gururaj, H. Hunag, C. Liu, G. Reinman, and Y. Zou, "An Energy-Efficient Adaptive Hybrid Cache," in Proc. ISLPED, 2011, pp. $67-72$.

[19] M. Qureshi, M. Franceschini, L. A. Lastras-Montaño, and J. Karidis, "Morphable Memory System: A Robust Architecture for Exploiting Multi-Level Phase Change Memories," in Proc. ISCA, 2010, pp. 153162.

[20] K. Tsuchida and et al., "A 64Mb MRAM with Clamped-Reference and Adequate-Reference Schemes," in Proc. ISSCC, 2010, pp. 258-259.

[21] D. H. Albonesi, "Selective Cache Ways: On-Demand Cache Resource Allocation," in Proc. MICRO, 1999, pp. 248-259.

[22] K. Shi and D. Howard, "Challenges in Sleep Transistor Design and Implementation in Low-Power Designs," in Proc. DAC, 2006, pp. 113116.

[23] P. Magnusson, M. Christensson, J. Eskilson, D. Forsgren, G. Hallberg, J. Hogberg, F. Larsson, A. Moestedt, and B. Werner, "Simics: A Full System Simulation Platform," in IEEE Computer, 2002, pp. 50-58.

[24] M. Martin, D. Sorin, B. Beckmann, M. Marty, M. Xu, A. Alameldeen, K. Moore, M. Hill, and D. Wood, "Multifacet's General ExecutionDriven Multiprocessor Simulator(GEMS) Toolset," in Computer Architecture News, 2005, pp. 92-99.

[25] CACTI 6.5, http://www.hpl.hp.com/research/cacti/.

[26] SPEC Benchmark, http://www.spec.org/cpu2006, 2006. 